\title{
Experimental and Numerical Study of Jet Noise Reflection and Shielding Effects by a Horizontal Tail ${ }^{*}$
}

\author{
Junichi AKATsuKA, ${ }^{1 \dagger}{ }^{\dagger}$ Kei WADA, ${ }^{2)}$ and Tatsuya IsHII ${ }^{3)}$ \\ ${ }^{1)}$ Aeronautical Technology Directorate, Japan Aerospace Exploration Agency, Mitaka, Tokyo 181-0013, Japan \\ ${ }^{2}$ Science Service Co., Ltd., Tokyo 101-0025, Japan \\ ${ }^{3)}$ Aeronautical Technology Directorate, Japan Aerospace Exploration Agency, Chofu, Tokyo 182-8522, Japan
}

\begin{abstract}
Experiments and numerical analyses were conducted to investigate the reflection and shielding effects of jet noise by a horizontal tail and an aft-mounted engine. An acoustic test using a cold jet at a Mach number of 0.98 was carried out with a 28.8-mm-diameter exhaust nozzle and a T-tail model. The effects of the position and angle of the horizontal tail were investigated using the boundary element method and a fitted jet noise source model. The experimental results showed that the sound pressure level increased by $1.5 \mathrm{~dB}$ in the downward direction of the aircraft at a Strouhal number of 0.94. The numerical analysis results showed that the reflection effect was highly dependent on the relative position and size of the horizontal tail, the location of the source, and the angle of the horizontal tail. In addition, the effect of the reflected direction was ascertained to find an appropriate horizontal tail and engine arrangement that reduces noise. It was found that the greatest influence in terms of the effective perceived noise level occurred when the reflected direction was $60^{\circ}$ from the jet axis.
\end{abstract}

Key Words: Jet, Noise, Compressible Flows, Experiments, Boundary Element Method

\section{Nomenclature}

\author{
$D$ : nozzle diameter \\ $f$ : frequency \\ $M_{\mathrm{j}}$ : jet Mach number \\ $r$ : radius \\ St: Strouhal number \\ $V_{\mathrm{j}}$ : jet velocity \\ $x, y, z$ : position \\ $\delta$ : horizontal tail angle \\ $\phi$ : azimuthal angle \\ $\theta$ : polar angle measured from jet axis
}

\section{Introduction}

Airport noise is one of the most important environmental issues associated with commercial aircraft. Because air traffic is expected to grow steadily in the future, ${ }^{1)}$ further noise reduction is required to promote the sustainability of air transportation. The International Civil Aviation Organization (ICAO) established a new noise standard for subsonic aircraft that applies to certification as of the end of 2017.2) New noise reduction technologies need to be developed to support such strengthened standards.

Jet noise is one of the largest components of takeoff and landing noise at airports, and it can be reduced significantly

(C) 2019 The Japan Society for Aeronautical and Space Sciences

*Partially presented at the 49th Fluid Dynamics Conference/35th Aerospace Numerical Simulation Symposium, 28-30 June 2017, Tokyo, Japan.

Received 6 October 2017; final revision received 9 September 2018; accepted for publication 16 October 2018.

†Corresponding author, akatsuka.junichi@jaxa.jp by increasing the engine bypass ratio of the aircraft. However, a large bypass ratio requires a large fan diameter, and this poses engine equipment issues. This is a particular problem for some types of aircraft such as business jets and future supersonic aircraft designs. A jet noise reduction technique that can serve as an alternative to increasing the engine bypass ratio is therefore needed for such types of aircraft.

These types of aircraft often have aft-fuselage-mounted engines with a T-tail. Burley et al. ${ }^{3)}$ noted that this configuration offers an advantage in terms of total system noise reduction. However, as researchers have also noted, jet noise reflection occurs because of T-tail use and could be a factor in reducing the benefit achieved. Isotani et al. ${ }^{4)}$ observed that the jet noise reflection that occurs with a T-tail is a sensitive issue in the design of supersonic aircraft because jet noise is the dominant source of airport noise for supersonic aircraft, whereas the tail volume affects other characteristics, such as stability and sonic boom. Therefore, the jet noise reflection and shielding effects associated with a T-tail configuration are among the key factors in reducing airport noise.

Many studies ${ }^{3-9)}$ have been conducted to examine the engine-airframe acoustic scattering problem, but most of such studies of interest examined noise reduction using engine noise shielding by the airframe. The combined effects of reflection and shielding have not been sufficiently investigated. Furthermore, the noise shielding characteristics of specific configurations such as T-tails have not yet been fully clarified.

This paper demonstrates trends in both jet noise reflection and shielding by a horizontal tail to simulate those effects for aircraft with aft-mounted engines and a T-tail. Scale-model experiments and the boundary element method (BEM) were used to investigate reflection and shielding effects. Based on the results, an appropriate arrangement for horizontal tail and 
nozzles that promote noise reduction are presented and discussed.

\section{Acoustic Test}

\subsection{Experimental setup}

Figure 1 shows the test model used in the acoustic test to investigate reflection and shielding effects. The model is based on the shape of the T-tail used in Japan Aerospace Exploration Agency (JAXA)'s silent supersonic research program. ${ }^{10)}$ The model consists of a T-tail and the right-side nozzle of twin engines mounted on a fuselage. The nozzle exit inner diameter is $28.8 \mathrm{~mm}$. The horizontal tail and vertical tail are simplified as plates. The thickness of the horizontal tail is $2.2 \mathrm{~mm}$, and that of the vertical tail is $2.4 \mathrm{~mm}$. The vertical tail is cut away at the line over the fuselage. The nozzle and core of the T-tail are made of plastic. The T-tail surface is formed from $0.2-\mathrm{mm}$-thick smooth aluminum plates. In the results presented in this paper, the origin of the coordinate system is defined as being at the center of the nozzle exit, and the orientations of the axes $(x, y, z)$ and angles $(\theta, \phi)$ are as shown in Fig. 1.

Acoustic tests were conducted at the JAXA noise test facility, ${ }^{11)}$ a schematic of which is shown in Fig. 2. This facility can produce a cold jet of pressurized air up to a jet Mach number of 1.8, adjusted by a control valve. In this test, the jet condition was set to a jet Mach number of 0.98 , and the total temperature ratio was set to 1 for all measurements. The test model was mounted vertically in an anechoic room $4.1 \mathrm{~m}$ wide, $5.7 \mathrm{~m}$ deep, and had a height of $3.3 \mathrm{~m}$. The room is equipped with a settling chamber on the floor. Far-field noise measurements were conducted with an array of eight Brüel \& Kjær 4939 1/4-inch microphones arranged in an arc $1.5 \mathrm{~m}(=52 D)$ from the nozzle exit, at polar angles $\theta$ from $20^{\circ}$ to $90^{\circ}$ at $10^{\circ}$ intervals. The microphones were calibrated before the test using a Brüel \& Kjær 4228 sound calibrator. The lower-limit frequency of the anechoic room was $400 \mathrm{~Hz}$. Acoustic data was sampled at $200 \mathrm{kHz}$ for $10 \mathrm{~s}$ through an $80 \mathrm{kHz}$ low-pass filter using a data logger (TEAC DS160R).

The estimated uncertainties in the $1 / 3$-octave band sound pressure levels (SPLs), including uncertainties associated with the instruments, jet conditions, duration, and sampling rate were $\pm 0.49 \mathrm{~dB}$ at $400 \mathrm{~Hz}$ (minimum frequency) and $\pm 0.37 \mathrm{~dB}$ at $63 \mathrm{kHz}$ (maximum frequency).

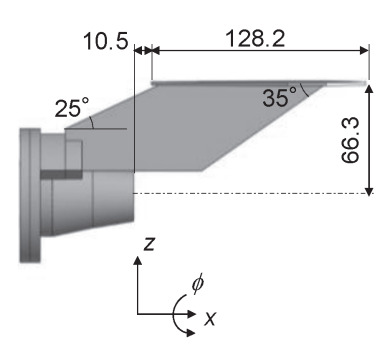

Side view

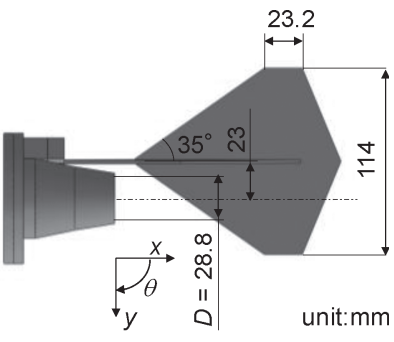

Bottom view
Fig. 1. Schematic of the test model.
The data was corrected for atmospheric absorption and microphone spectral response based on information provided by the supplier (Brüel \& Kjær). In this study, frequency $f$ was normalized with the nozzle diameter $D$ and the jet velocity $V_{\mathrm{j}}$ as the Strouhal number $S t=f D / V_{\mathrm{j}}$, and the SPL spectrum was sought on unit $S t$ at the measured position.

\subsection{Experimental results}

Figure 3 shows the data measured in the vertical direction. In Fig. 3, $\phi=0^{\circ}$ represents the shielded side ( $+z$ direction), whereas $\phi=180^{\circ}$ represents the reflection side $(-z$ direction). Polar angles $\theta$ of $20^{\circ}$ and $90^{\circ}$ are shown here. Jet noise reflection and shielding effects were observed around $\theta=90^{\circ}$ and $S t>0.1$. A significant change was observed at a high frequency. On the shielded side, the difference between the conditions with and without the T-tail gradually increased as the frequency increased, whereas the maximum reflection effect was observed around $S t=1$ on the reflection side. The differences at $S t=0.94$ were $1.6 \mathrm{~dB}$ on the shielded side and $1.5 \mathrm{~dB}$ on the reflected side.

Figure 4 shows the results in the horizontal direction $\left(\phi=90^{\circ}, 270^{\circ}\right)$. A difference with and without the T-tail was not observed in the horizontal direction. These results in-

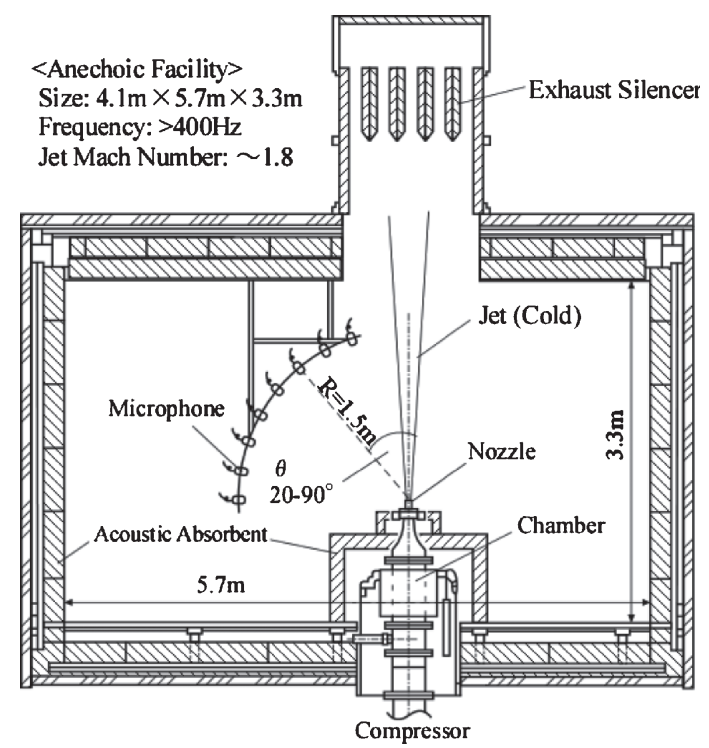

Fig. 2. Noise test facility at JAXA.

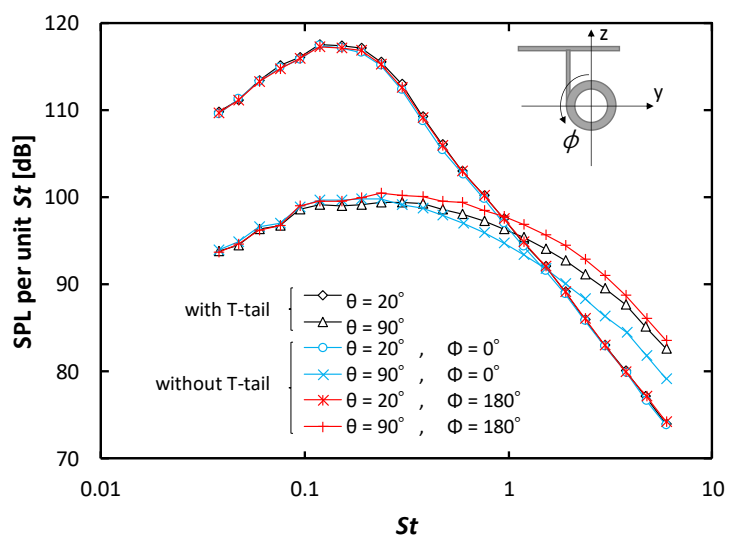

Fig. 3. Experimental results (vertical direction). 


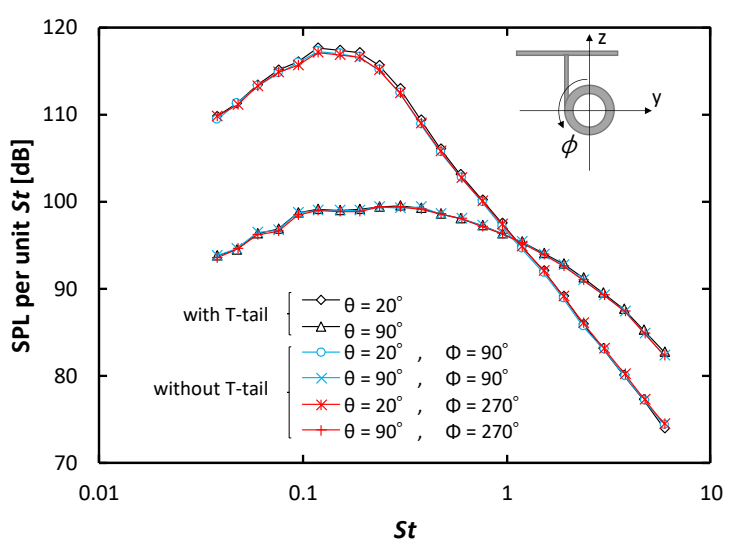

Fig. 4. Experimental results (horizontal direction).

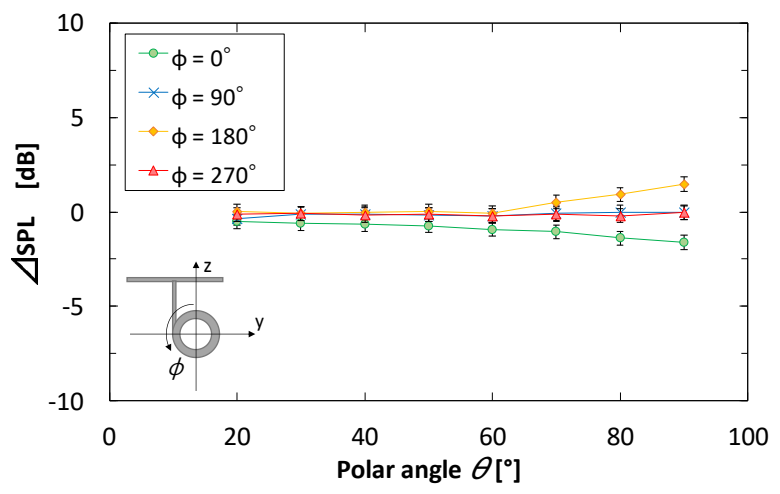

Fig. 5. SPL difference with and without T-tail at a St of 0.94 .

dicate that the vertical tail shown in Fig. 1 affects neither jet noise reflection nor shielding.

Figure 5 shows the SPL difference with and without the Ttail as a function of the polar angle at a $S t$ of $0.94(10 \mathrm{kHz})$, which is a frequency at which both large reflection and shielding effects are observed. The difference on the reflection side increased from $\theta=60^{\circ}$ to $\theta=90^{\circ}$. The maximum difference on the shielded side was also observed to occur at $\theta=90^{\circ}$. However, the difference decreased more gradually at smaller polar angles.

\section{Numerical Analysis}

The experimental results clearly showed that the horizontal tail of the T-tail affect jet noise reflection and shielding in the vertical direction. Consequently, numerical analyses were conducted to investigate the effects of the arrangement of the horizontal tail.

\subsection{Numerical method}

The boundary element method (BEM) was used for numerical analysis. BEM has often been applied to analyze the acoustic scattering problem. The sound pressure in a stationary medium at point $\boldsymbol{r}_{\mathrm{p}}, p\left(\boldsymbol{r}_{\mathrm{p}}\right)$, was described using the following Kirchhoff-Helmholtz equation ${ }^{12)}$ with boundary $\Gamma$ :

$\varepsilon p\left(\boldsymbol{r}_{\mathrm{p}}\right)=q\left(\boldsymbol{r}_{\mathrm{p}}\right)-\int_{\Gamma}\left(p(\boldsymbol{r}) \frac{\partial G\left(\boldsymbol{r}_{\mathrm{p}}, \boldsymbol{r}\right)}{\partial n}-G\left(\boldsymbol{r}_{\mathrm{p}}, \boldsymbol{r}\right) \frac{\partial p(\boldsymbol{r})}{\partial n}\right) d \Gamma$ where the constant is $\varepsilon=1$ in the exterior of $\Gamma, 0$ in the interior of $\Gamma$, and 0.5 on $\Gamma$ if $\Gamma$ is smooth around $\boldsymbol{r}_{\mathrm{p}} ; q\left(\boldsymbol{r}_{\mathrm{p}}\right)$ is the incident acoustic pressure; and $G$ is the three-dimensional free-space Green function given by the following:

$$
G\left(\boldsymbol{r}_{\mathrm{p}}, \boldsymbol{r}\right)=\frac{\exp \left(i k\left|\boldsymbol{r}_{\mathrm{p}}-\boldsymbol{r}\right|\right)}{4 \pi\left|\boldsymbol{r}_{\mathrm{p}}-\boldsymbol{r}\right|}
$$

Because Eq. (1) gives a scattered acoustic field for a given incident acoustic field, a model of a jet noise source as an incident acoustic field is also important. Some previous studies $^{5,9,13,14)}$ have noted that there are difficulties associated with expressing jet noise using a point source in acoustic scattering problems. Jet noise modeling via an analytical approach has been conducted by Tam and Burton, ${ }^{15)}$ Morris, ${ }^{16)}$ and Reba et al. ${ }^{17)}$ Recently, Papamoschou ${ }^{9)}$ proposed a new jet noise modeling method using the combination of a wave packet formulation $p_{\mathrm{w}}$ and a point source $p_{\mathrm{p}}$. This combination is expressed as follows:

Wave packet model:

$$
p_{\mathrm{w}}=p_{0}(x) e^{(-i \omega t+i m \varphi)}
$$

where

$$
p_{0}(x)=\tanh \left(x / b_{1}\right)^{p_{1}}\left\{1-\tanh \left(x / b_{2}\right)^{p_{2}}\right\} e^{i \propto x}
$$

and

Point source model:

$$
p_{\mathrm{p}}=\frac{Q}{4 \pi r} e^{(-i \omega t+i k r)}
$$

This combined model requires seven model constants $\left(m, b_{1}, b_{2}, p_{1}, p_{2}, \alpha\right.$, and $Q$ ) and a source origin $x_{0}$. In this study, the seven model constants were determined so that far-field noise produced by the model matched the experimental data without a T-tail, based on the original study. ${ }^{9)} x_{0}$ was determined experimentally according to the peak noise location for each frequency using a noise source map obtained by a phased-array-microphone system in the original study. ${ }^{9)}$ However, because using his noise source map was not appropriate for our experimental condition, in this study, $x_{0}$ was estimated using the empirical model proposed by Brown $^{18)}$ assuming the jet potential core length was $6.8 D$ from an additional CFD analysis, which resulted in $x_{0}=5 \mathrm{D}$. Figure 6 shows the model constants and a comparison of the model and experimental data at $52 D$ from the nozzle exit. This figure shows the zero-shifted SPL data using the reference data, $\operatorname{SPL}_{\mathrm{ref}}\left(\theta=20^{\circ}, r=52 D\right)$. The figure shows that the far-field noise predicted by the model agrees reasonably well with the data measured. Figure 7 shows an incident acoustic field generated by the model at the nozzle center plane $(y=0)$. The values are also zero-shifted using SPL $\mathrm{Sef}_{\text {, }}$ and the values inside the jet are not shown because they are not given in the model. ${ }^{9)}$ The contour shows that the model can generate the directivity of jet noise that has the maximum SPL around $\theta=40^{\circ}$. The numerical results of the following parts will be shown as the scattered field based on this incident acoustic field.

A parametric study on the arrangement of the horizontal 


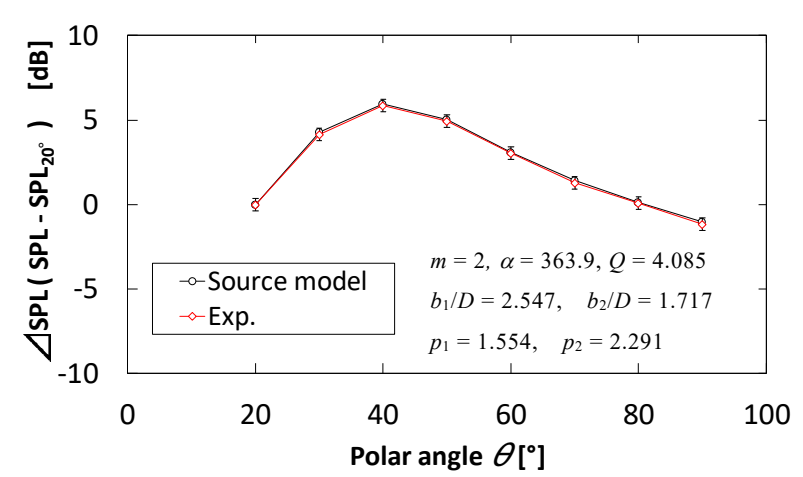

Fig. 6. Comparison of jet noise source model and experimental data for SPL at $52 D$ from the nozzle exit.

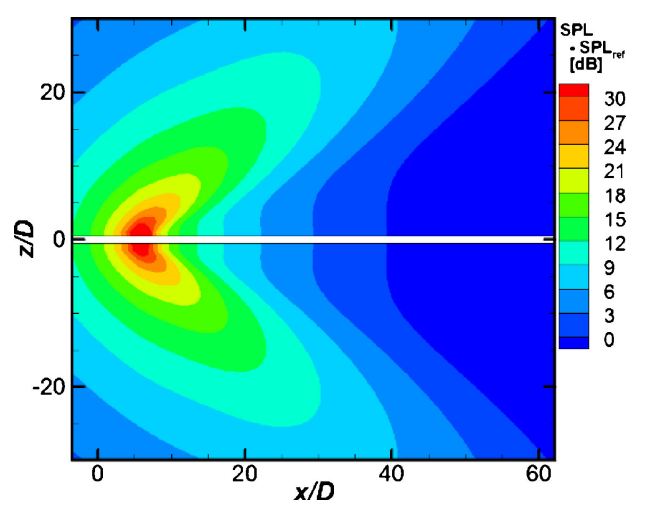

Fig. 7. Incident acoustic field $(S t=0.94)$.

tail conducted using BEM was performed for $S t=0.94$, which was examined in Fig. 5. Because the jet noise reflection and shielding effects caused by the horizontal tail appeared to be more sensitive than those by the vertical tail, in terms of noise propagation to the ground as seen in the experiments, the analyses discussed in this paper focus on the effects of the horizontal tail. Therefore, only the horizontal tail was discretized. This was done using 6,604 triangular elements, the length of each of which was less than $1 / 8$ of the wavelength. A BEM code developed at JAXA was used. This code was validated with respect to the exact solution for acoustic scattering from a rigid sphere. ${ }^{19)}$ The boundary surface was treated as a rigid wall. The parameters considered in the numerical analysis are shown in Table 1 . The arrangement of the horizontal tail, the horizontal tail angle, and the size of the horizontal tail were examined.

\subsection{Numerical results and discussion}

Figure 8 compares the numerical results with the acoustic data measured for Case 0 (the baseline). The trends in the predicted jet noise reflection and shielding effect agree reasonably well with the data measured. The predicted values are slightly greater than the values measured. At $\theta=90^{\circ}$, the reflection effect was over-predicted by $0.5 \mathrm{~dB}$, and the shielding effect was over-predicted by $2 \mathrm{~dB}$. The scattered acoustic field at the nozzle center plane $(y=0)$ obtained using BEM for Case 0 is illustrated in Fig. 9. The increase in SPL in the downward direction of the horizontal tail and decrease in SPL behind the horizontal tail are both significant, as seen in Fig. 9.
Table 1. Parameters considered in the numerical analysis.

\begin{tabular}{clcc}
\hline Case ID & $\begin{array}{c}\text { Horizontal } \\
\text { tail position }\end{array}$ & $\begin{array}{c}\text { Horizontal } \\
\text { tail angle } \delta\end{array}$ & $\begin{array}{c}\text { Horizontal tail } \\
\text { surface area }\end{array}$ \\
\hline Case 0 & Baseline & $0^{\circ}$ & Baseline \\
Case 1 & $1 D$ forward & $0^{\circ}$ & Baseline \\
Case 2 & $1 D$ rearward & $0^{\circ}$ & Baseline \\
Case 3 & $1 D$ upper side & $0^{\circ}$ & Baseline \\
Case 4 & $1 D$ down side & $0^{\circ}$ & Baseline \\
Case 5 & Baseline & $-5^{\circ}$ & Baseline \\
Case 6 & Baseline & $+5^{\circ}$ & Baseline \\
Case 7 & Baseline & $0^{\circ}$ & Doubled \\
\hline
\end{tabular}

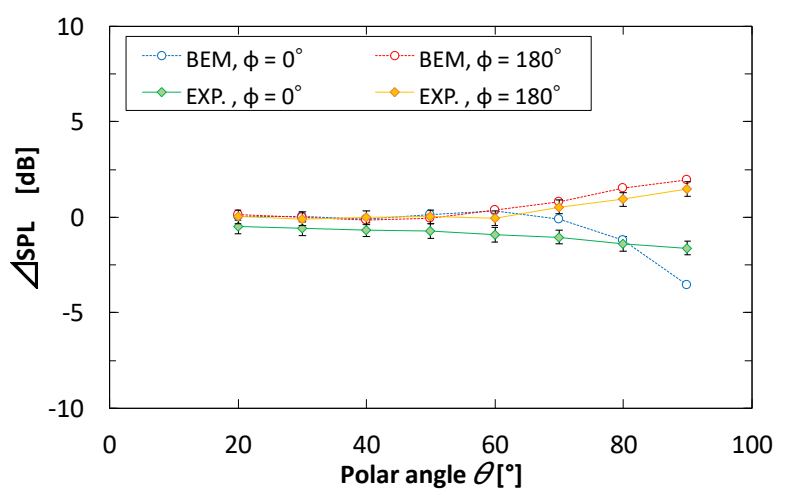

Fig. 8. Comparison of experimental results with numerical analyses (Case $0)$.

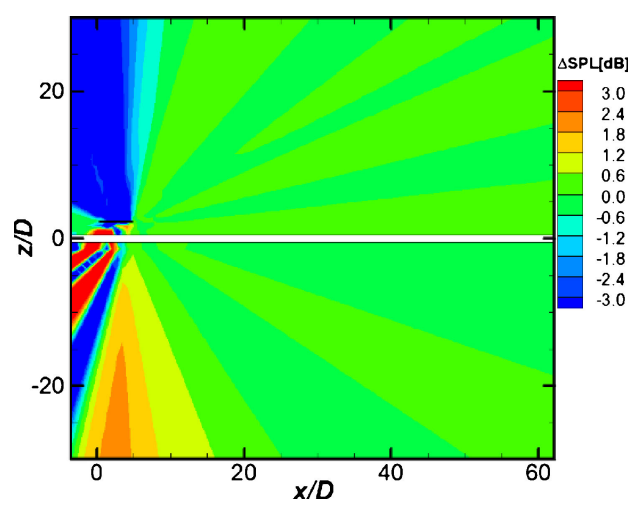

Fig. 9. Scattered acoustic field for $y=0$ (Case 0$)$.

The numerical results for changes in the arrangement of the horizontal tail (Cases 1-4) are shown in Figs. 10(a)(d). Figures 10(a) and (b) show that the horizontal tail position in the $x$ direction is sensitive to jet noise reflection and shielding effects. Figures 10(c) and (d) show that variation in the effects caused by changing the position of the horizontal tail in the $z$ direction is more moderate. Among Cases $0-4$, Case 3 (the upper position) is the best for reducing the reflection effect in the downward direction.

The scattered acoustic fields at the nozzle center plane $(y=0)$ are shown in Figs. 11(a)-(d) for Cases 1-4. Each figure supports the results described above. Figures 11(a) and (b) indicate that the variation caused by the position in the $x$ direction depends on the size of the horizontal tail area covering the incident acoustic wave, which depends on the relative distance between the jet noise source and the horizontal tail. On the other hand, the variation produced by 


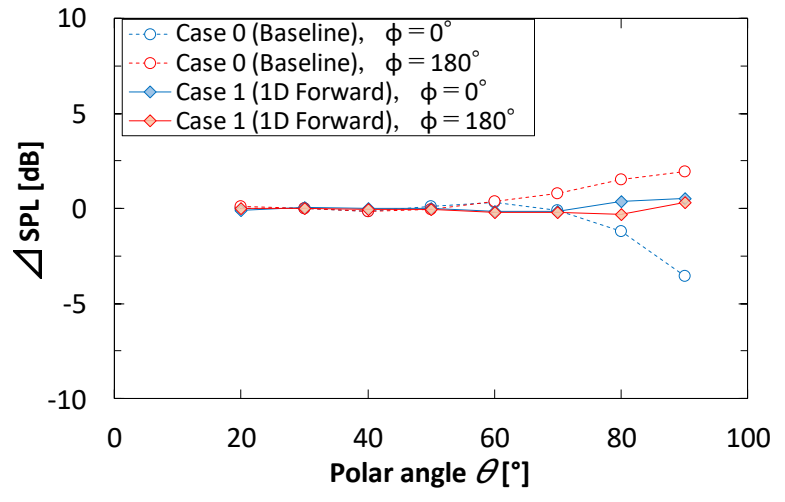

(a) Case 1

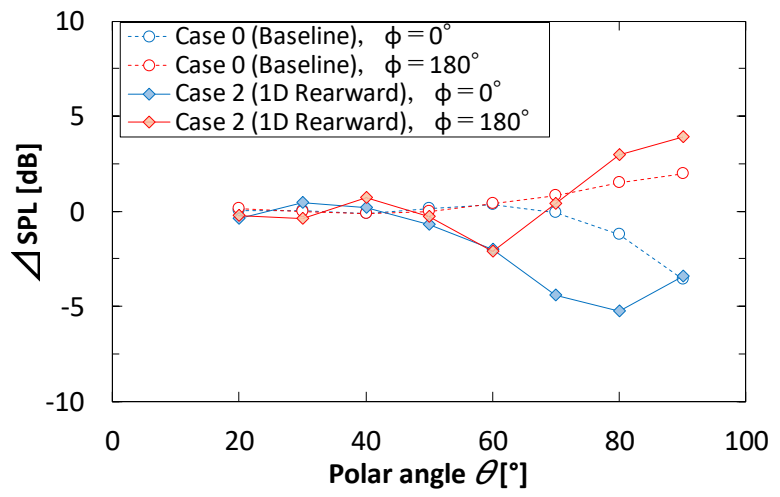

(b) Case 2

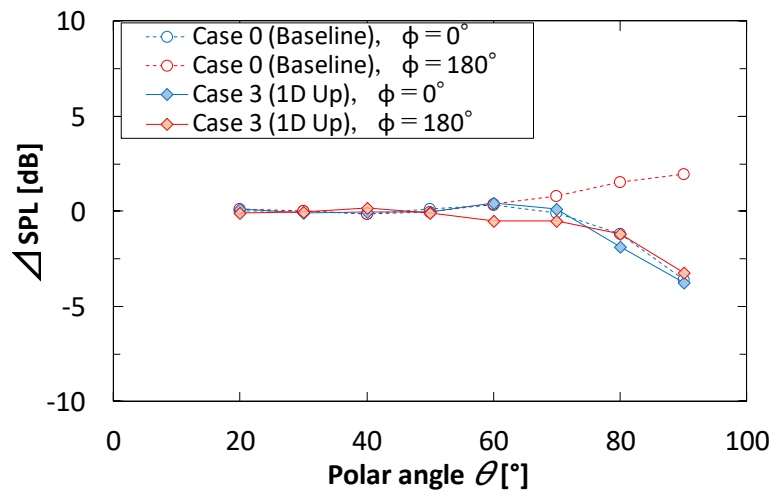

(c) Case 3

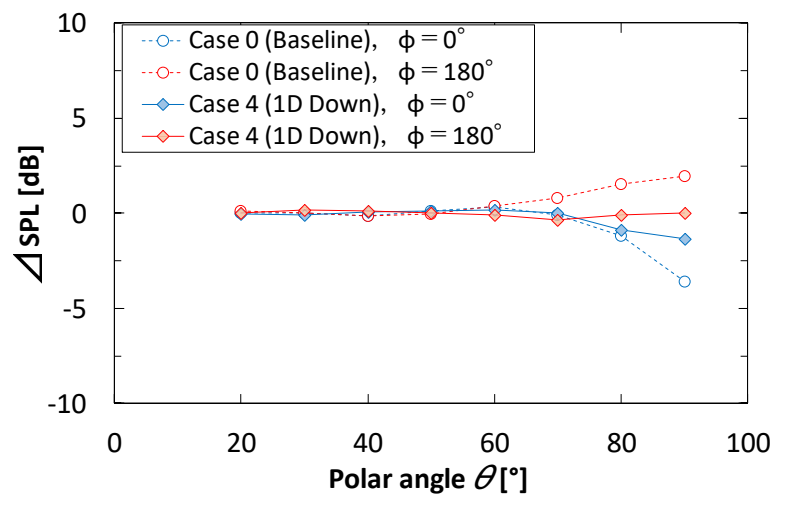

(d) Case 4

Fig. 10. Numerical results.

changing the vertical position seems to be caused by the difference in the reflected direction induced by the horizontal tail, according to the scattering patterns in Figs. 11(c) and (d).

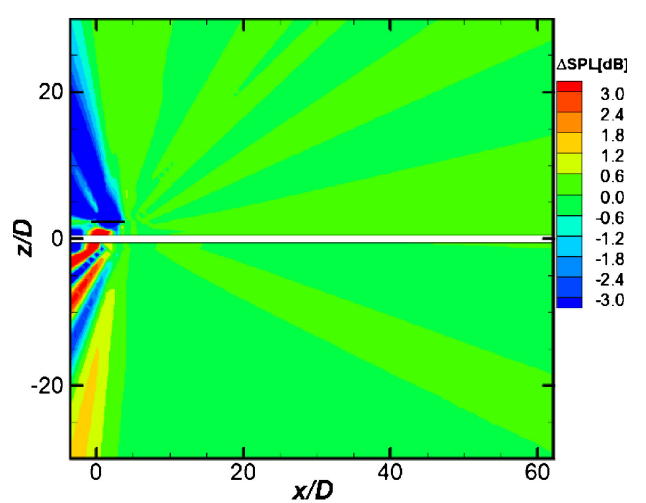

(a) Case 1

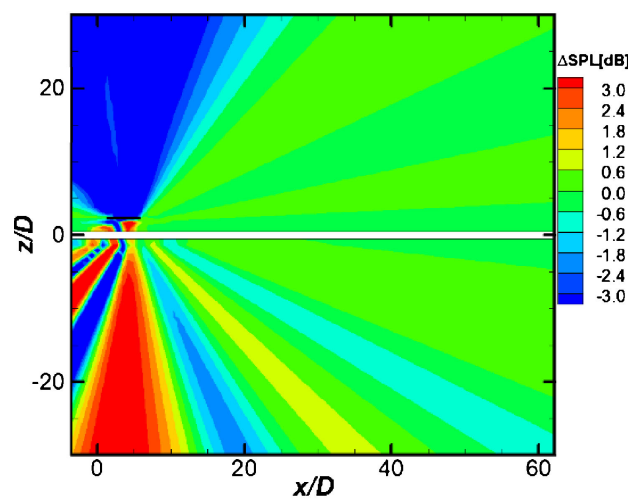

(b) Case 2

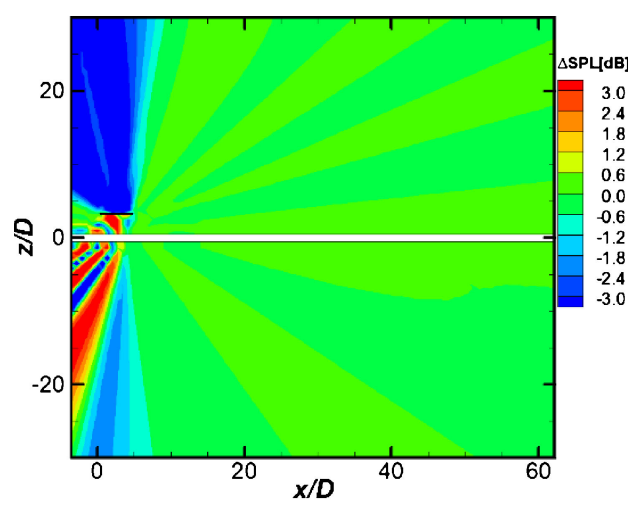

(c) Case 3

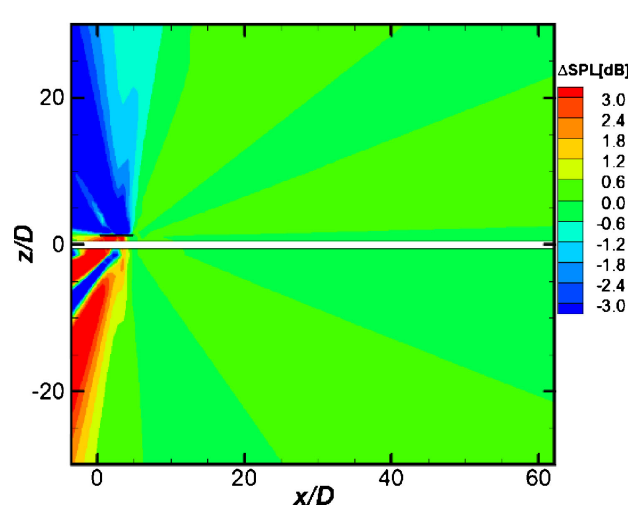

(d) Case 4

Fig. 11. Scattered acoustic field for $y=0$.

The effects of the horizontal tail angle are shown in Figs. 12(a) and (b). The reflection effect varied with the horizontal tail angle $\delta$, whereas the shielding effect did not vary. 


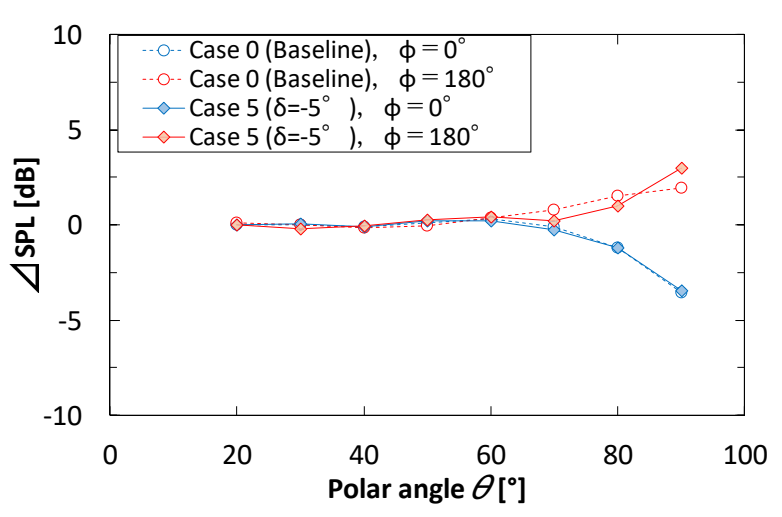

(a) Case 5

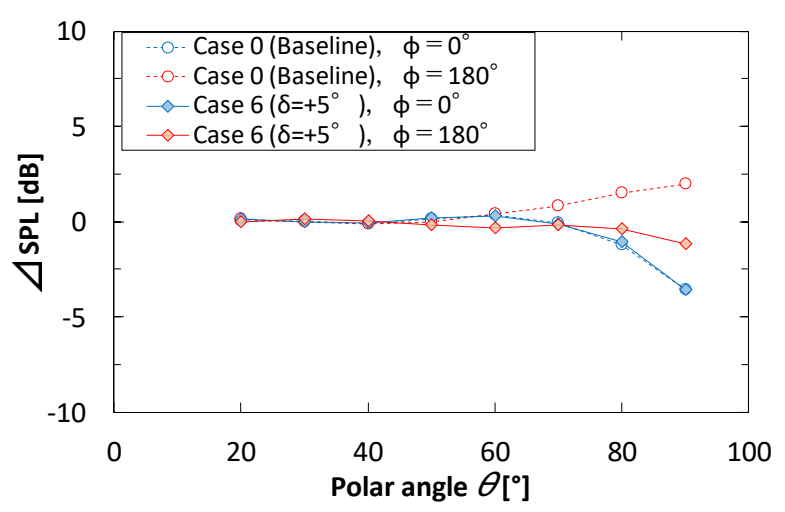

(b) Case 6

Fig. 12. Numerical results.

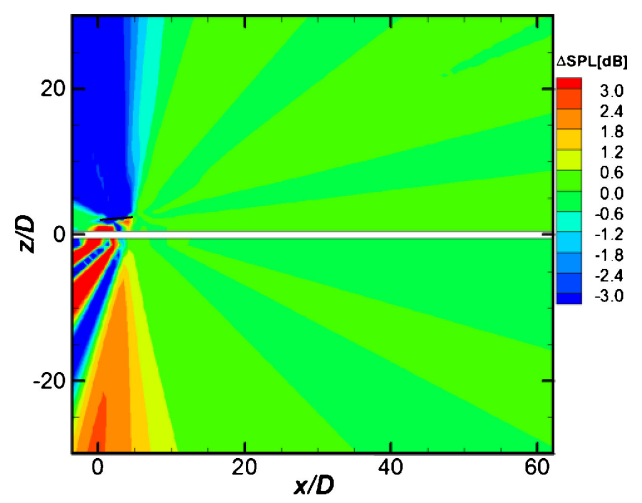

(a) Case 5

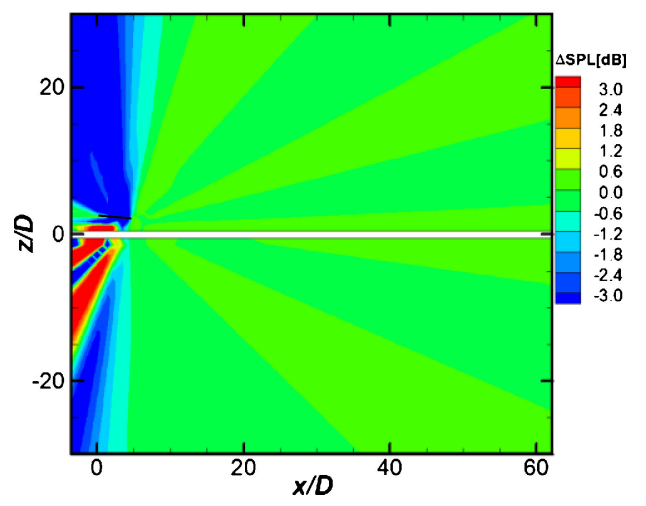

(b) Case 6

Fig. 13. Scattered acoustic field for $y=0$.

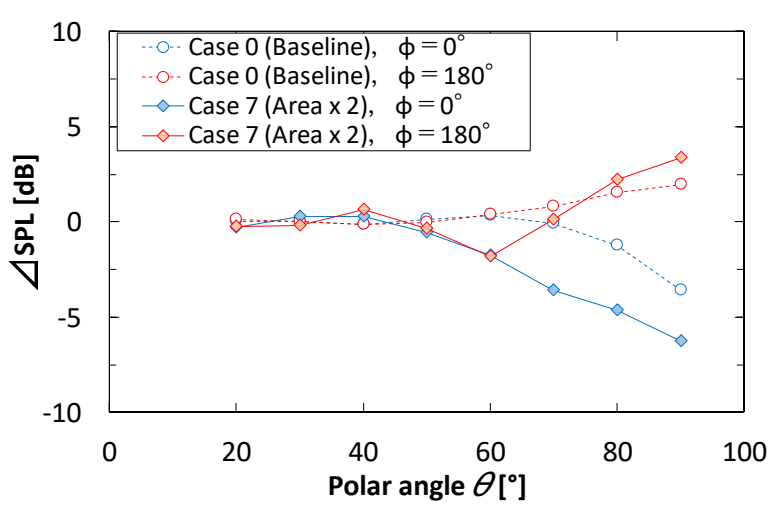

Fig. 14. Numerical results for Case 7.

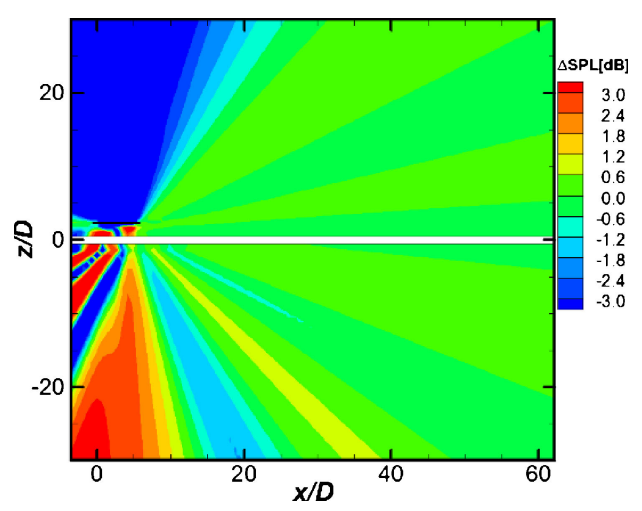

Fig. 15. Scattered acoustic field for $y=0$ (Case 7).

These results indicate that the reflection effect is sensitive to the incident angle, whereas the shielding effect is mainly affected by the size of the horizontal tail area covering the incident acoustic wave.

Figures 13(a) and (b) show the scattered acoustic fields for Cases 5 and 6. A significant change in the difference of SPL around $\theta=90^{\circ}$ on the reflection side can be observed when the horizontal tail angle is changed. This indicates that the horizontal tail angle is also an important factor relating to reflection.

Figure 14 shows the results for Case 7, which examined a doubled horizontal tail area. The results show that both reflection and shielding effects are increased.

Figure 15 shows the scattered acoustic field for Case 7. The counter clearly shows an increase in the affected area as horizontal tail area increases.

\section{Discussion on Horizontal Tail Arrangement for Noise Reduction}

The experimental data showed that a horizontal tail has a significant effect on jet noise reflection and shielding, and the numerical analysis results showed that the reflection effect depends on the incident and reflected angles, as well as the size of the horizontal tail. These results naturally lead to the question of which direction has a more serious effect on airport noise when reflection occurs. To investigate the sensitivity of the reflected direction to airport noise, a simu- 


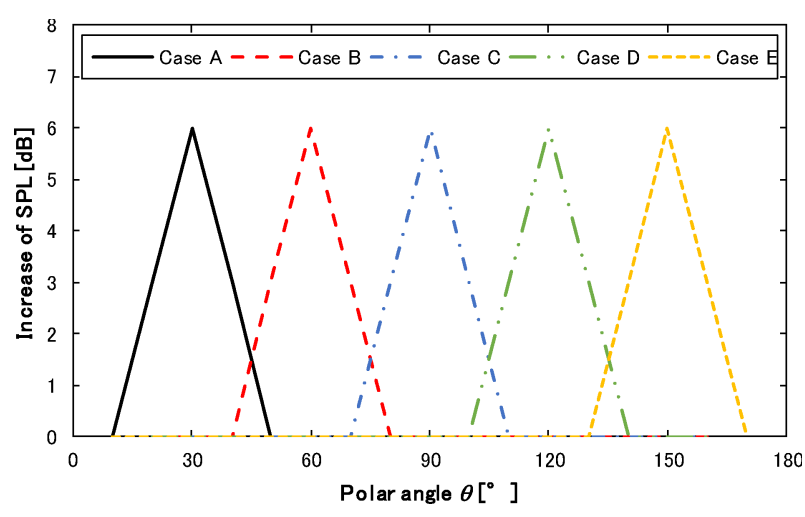

Fig. 16. Simulation model for increase in SPL due to reflection effect.

lation was conducted using JAXA's noise estimation code, AiNEST. ${ }^{20)}$ The code was developed to simulate aircraft takeoff and landing noise, including the effects of aircraft configuration, flight trajectory, and metrics of the noise standard. ${ }^{2)}$ The code can simulate noise using empirical noise source and propagation models. In the simulation, level flight at an altitude of $609.6 \mathrm{~m}$, a true air speed of $100 \mathrm{~m} / \mathrm{s}$, a jet velocity of $320 \mathrm{~m} / \mathrm{s}$, and a nozzle diameter of $1 \mathrm{~m}$ were assumed. Only the jet noise component, which includes the forward flight effect, was taken into account in estimating the airport noise. The data was evaluated at a measurement point under the flight profile in terms of an effective perceived noise level (EPNL). The details of the airport noise standard are found in Annex 16 Environmental Protection Volume I Aircraft Noise. ${ }^{2)}$ The jet noise directivity in the jet noise source model was varied to simulate an increase in SPL due to the reflection effect. The increase in SPL was simply modeled using the function shown in Fig. 16.

The results of the simulation are given in Table 2 . The results indicate that the strongest impact, in terms of EPNL, occurred for the case in which a SPL of $60^{\circ}$ is increased. This is the result of jet noise directivity itself, and of the distance between the noise source and the measurement point on the ground. Therefore, the results indicate that the arrangement of the engine and horizontal tail should not lead to reflection toward the aft side around $\theta=60^{\circ}$ of the aircraft, even if jet noise reflection is unavoidable.

In the example of analyses described in the previous section, the horizontal tail arrangements seen in Cases 1, 3, and 6 are preferred to the initial position (Case 0) because these positions reduce interference between jet noise radiation and the horizontal tail or weaken the jet noise reflection toward the aft side. In contrast, Cases 2 and 7, which strengthen interference, are not desired. As shown in the analyses, it is desirable to consider the horizontal tail arrangement as well as its aerodynamic performance for jet noise reflection for the purpose of realizing quieter aircraft.

\section{Conclusions}

Acoustic tests for a scale model and numerical analyses using the boundary element method were conducted to in-
Table 2. Effect of reflected direction

\begin{tabular}{ccc}
\hline Case ID & $\begin{array}{c}\text { Polar angle of } \\
\text { increased SPL, }\end{array}$ & $\begin{array}{c}\text { Difference from scenario } \\
\text { without a horizontal tail } \\
\text { in EPNL, dB }\end{array}$ \\
\hline Case A & 30 & 0.6 \\
Case B & 60 & 2.4 \\
Case C & 90 & 1.6 \\
Case D & 120 & 1.0 \\
Case E & 150 & 0.4 \\
\hline
\end{tabular}

vestigate trends in both jet noise reflection and the shielding effects of the horizontal section of a T-tail for a cold jet at a Mach number of 0.98 . The results of this study are summarized as follows:

(1) The jet noise reflection and shielding effects of a horizontal tail were observed at approximately $\theta=90^{\circ}$ and $S t>0.1$ during the acoustic test. A significant change was observed at a high frequency. On the shielded side, the difference between the conditions with and without the T-tail gradually increased as the frequency increased, whereas the maximum reflection effect was observed at approximately $S t=1$ on the reflection side. The differences at $S t=0.94$ were $1.6 \mathrm{~dB}$ on the shielded side and $1.5 \mathrm{~dB}$ on the reflected side.

(2) Numerical analyses revealed that the reflection effect of a horizontal tail is sensitive to both the size of the horizontal tail area covering the incident acoustic wave and the incident angle, whereas the shielding effect mainly depends on the size of the horizontal tail area.

(3) An investigation of the influence resulting from reflection direction on the effective perceived noise level using an empirical noise estimation code showed that a reflection to $60^{\circ}$ from the jet axis direction has the strongest effect. Therefore, if jet noise reflection by a horizontal tail is not avoidable, the direction of reflection should not be toward the aft side of the aircraft at approximately $\theta=60^{\circ}$.

In this study, we examined the jet noise reflection and shielding effects for a cold jet under static conditions. In the future, studies on a hot jet including the forward flight effect will be conducted.

\section{Acknowledgments}

The authors would like to thank H. Obata of the Tokyo University of Agriculture and Technology and T. Wada of Campus Create for their contributions to the experiments.

\section{References}

1) Japan Aircraft Development Corporation: Worldwide Market Forecast 2017-2036, Japan Aircraft Development Corporation, Tokyo, 2017.

2) International Civil Aviation Organization: Annex 16 Environmental Protection Volume I Aircraft Noise, 7th ed., Montreal, 2014.

3) Burley, C. L., Thomas, R. H., and Guo, Y.: Quantification of Acoustic Scattering Prediction Uncertainty for Aircraft System Noise Assessment, AIAA Paper 2016-3041, 2016.

4) Isotani, K., Hayama, K., Makino, Y., and Akatsuka, J.: Study on the Shielding Effect with Tails for Noise Reduction of a Small Supersonic Transport, Proceedings of JSASS Aircraft Symposium, Oct. 2015 (in Japanese).

5) Hayama, K., Fukata, S., and Azuma, T.: Analytical Estimation of Jet 
Noise Source Shielding Effect for Next SST, Proceedings of JSASS Aircraft Symposium, Nov. 2012 (in Japanese).

6) Hattori, Y. and Imamura, T.: A Estimation of Engine Noise Reflection and Shielding around Aircraft using Ray Tracing, The Transportation and Logistics Conference 2014(23), 2014, pp. 177-180 (in Japanese).

7) Doty, M. J., Brooks, F., Burley, C. L., Bahr, C. J., and Pope, D. S.: Jet Noise Shielding Provided by a Hybrid Wing Body Aircraft, AIAA Paper 2014-2625, 2014.

8) Papamoschou, D. and Mayoral, S.: Jet Noise Shielding for Advanced Hybrid Wing-Body Configuration, AIAA Paper 2011-0912, 2011.

9) Papamoschou, D.: Prediction of Jet Noise Shielding, AIAA Paper 2010-065, 2010.

10) D-SEND Project/Silent Supersonic Transport Technology, http: //www.aero.jaxa.jp/eng/research/frontier/sst/ (accessed September 20, 2017).

11) Ishii, T., Yamamoto, K., Nagai, K., and Ishii, Y.: Overview of Jet Noise Measurement at JAXA, Trans. JSASS Aerospace Technology Japan, 12, APSAT2013 (2014), pp. 123-132..

12) Lu, Y.: Fast Multipole Boundary Element Method, Cambridge University Press, London, 2009, pp. 152-154.

13) Papamoschou, D. and Mayoral, S.: Modeling of Jet Noise Sources and Their Diffraction with Uniform Flow, AIAA Paper 2013-0326, 2013.
14) Papamoschou, D.: Wavepacket Modeling of the Jet Noise Source, AIAA Paper 2011-2835, 2011

15) Tam, C. K. W. and Burton, D. E.: Sound Generation by the Instability Waves of Supersonic Flows. Part 2. Axisymmetric Jets, J. Fluid Mech., 138 (1984), pp. 273-295.

16) Morris, P. J.: Jet Noise Prediction: Past Present and Future, Can. Acoustics, 35, 3 (2007), pp. 16-22.

17) Reba, R., Narayanan, S., and Colonius, T.: Wave-Packet Models for Large-Scale Mixing Noise, Int. J. Aeroacoustics, 9, 4-5 (2010), pp. 533-557..

18) Brown, C.: Empirical Models for the Shielding and Reflection of Jet Mixing Noise by a Surface, NASA-TM2016-218914, 2016.

19) Crighton, D. G., Dowling, A. P., Ffowcs-Williams, J. E., and Heckl, M.: Modern Methods in Analytical Acoustics Lecture Notes, Springer, New York, 1992, pp. 533-535.

20) Akatsuka, J.: Development of Aircraft Noise Estimation Tool (AiNEST), JAXA-RR-16-005, 2017 (in Japanese).

Naofumi Ohnishi Associate Editor 